\title{
Parasitemia and its daily variation in canine leishmaniasis
}

\author{
Simona Di Pietro ${ }^{1}$ (D) $\cdot$ Chiara Crinò $^{1} \cdot$ Annastella Falcone ${ }^{1} \cdot$ Rosalia Crupi $^{1} \cdot$ Francesco Francaviglia $^{2} \cdot$ Fabrizio Vitale $^{3}$. \\ Elisabetta Giudice ${ }^{1}$
}

Received: 11 September 2019 / Accepted: 5 August 2020 / Published online: 15 August 2020

(C) Springer-Verlag GmbH Germany, part of Springer Nature 2020

\begin{abstract}
The aim of this study was to evaluate, through qPCR, the prevalence of parasitemia in sick kennel dogs naturally infected by canine leishmaniasis. An evaluation of daily changes of the parasitic load in peripheral blood was also performed. A comprehensive clinical examination and the collection of several samples (blood, lymph node, skin, and conjunctiva) were performed in 140 dogs living in an endemic area. Among these, only the dogs with clinically evident leishmaniasis were enrolled (39/140; $27.9 \%$ ). Twelve (30.8\%) out of 39 showed parasitemia, with a low load (median: 4 Leishmania $/ \mathrm{ml}$ ) despite a high lymph node parasite load (median: 4000 Leishmania $/ \mathrm{ml}$ ) and high IFAT titers $(\geq 1: 640)$. Seven sick dogs were sampled every $4 \mathrm{~h}$ for 6 times during a 24-h period, in order to obtain light- and dark-span samples. Only one (14.3\%) out of the seven serial sampled dogs showed Leishmania DNA in the peripheral blood in two samples (2/42;4.8\%). Surprisingly, Leishmania DNA was also detected in the peripheral blood of asymptomatic dogs, negative to both serology and PCR performed on samples other than blood (6/101; $5.9 \%)$. The present study confirms that in canine leishmaniasis parasitemia is uncommon and even transitory. Even if recommended, microscopic examination is confirmed as a low sensitivity method with a lower diagnostic utility in canine leishmaniasis than qPCR. Moreover, circulating Leishmania DNA can be found even in healthy dogs. This finding is important in clinical practice because in endemic areas it suggests a transfusion risk and a possible transmission to the vector.
\end{abstract}

Keywords Amastigote and promastigote forms $\cdot$ Daily variation of parasitemia $\cdot$ Dog $\cdot$ Leishmania infantum $\cdot \mathrm{qPCR}$

\section{Introduction}

Canine leishmaniasis (CanL), caused by Leishmania infantum, is an important zoonotic vector-borne disease, endemic in many countries all over the world (Alvar et al. 2012). In the past 10 years, CanL is of increasing concern in many regions of the Mediterranean basin, including Italy, since it spreads in previously non-endemic areas in relationship to the sandfly vectors (Mendoza-Roldan et al. 2020). Leishmania spp. are digenetic parasites, with a life cycle involving two

Section Editor: Domenico Otranto

Simona Di Pietro

dipietros@unime.it

1 Department of Veterinary Sciences, University of Messina, Polo Universitario dell'Annunziata, 98168 Messina, Italy

2 Local Animal Health Veterinarian, ASP (Azienda Sanitaria Provinciale) Palermo, Palermo, Italy

3 Istituto Zooprofilattico Sperimentale della Sicilia “A. Mirri”, Via Rocco Dicillo 9, 90139 Palermo, Italy hosts, a vertebrate, and the invertebrate host-sandfly (Phleobotomus spp. and Lutzomyia spp.). During blood meal from an infected hosts, a female sandfly injects promastigotes that are phagocytized by the monocytes/macrophages and transform into intracellular amastigotes. Although Leishmania amastigotes are typically phagocytized by macrophages in peripheral blood, they are seen engulfed primarily by neutrophils, supporting the theory that neutrophils are used as carriers enabling the "silent entry" of the protozoa into macrophages ("Trojan horse" theory) (Oikonomidis et al. 2019). Several diagnostic tools have been applied to detect Leishmania infection in dogs, including parasitological, serological, and molecular techniques. Molecular diagnostic tests, notably real-time polymerase chain reaction (qPCR), are highly sensitive and specific tests for the diagnosis of CanL and the monitoring parasite loads in different biological samples (Ramos et al. 2013). The duration, constancy, and intensity of parasitemia in canine host are still largely unknown leading to false negatives especially in asymptomatic dogs. False positives can also occur due to a transient infection (Maia and Campino 2008). In human L. infantum infection, the presence of amastigote forms in peripheral blood is considered a rare or 
occasional finding in immunocompetent individuals (Chemli et al. 2006). Conversely, the parasitemia is more frequent during human Kala-azar by L. donovani in India and East Africa (Anand et al. 2004; Saran et al. 1997; Nandy 1986) and in immunocompromised patients (Izri et al. 1996), with a peak parasite load in blood during the night (Sharma et al. 2000; Saran et al. 1997). Furthermore, the presence of the parasite was seen in monocytes and neutrophils on an affected Italian man's peripheral blood smears (Fiorini et al. 2002). In veterinary medicine, parasites were rarely detected in blood smears. In our previous study, amastigotes were found in only four $(0.3 \%)$ out of 1438 leishmaniotic dogs (Giudice and Passantino 2011), both free and inside circulating leukocytes (neutrophil, monocyte, macrophage). All the dogs found with parasitemia were severely ill, and three of them had concomitant ehrlichiosis. Similarly, the presence of several amastigotes, free or in circulating neutrophils, was observed in a dog co-infected with Ehrlichia canis (Foglia Manzillo et al. 2005) or with Dirofilaria spp. (Oikonomidis et al. 2019). In a severely sick dog affected by both leishmaniasis and babesiosis, many amastigotes included in macrophages and a parasite on circulating monocyte were found in ascitic fluid and peripheral blood, respectively (Ruiz de Gopegui and Espada 1998). The presence of parasites in the blood is now widely recognized as a potential risk for transmission of visceral leishmaniasis through blood transfusions, both in man and in dogs (Riera et al. 2008; Tabar et al. 2008; de Freitas et al. 2006; Kyriakou et al. 2003; Otero et al. 2000). The fact that even asymptomatic individuals can transmit the infection has important clinical implication. In a few studies carried out in North America on dogs undergoing blood transfusion, L. infantum has been transmitted by infected donor dogs (Giger et al. 2002; Owens et al. 2001). In a molecular (PCR) screening carried out in a blood bank which used dog donors coming from endemic areas (Barcelona, Spain), L. infantum DNA was isolated in the $20 \%$ of the samples (Tabar et al. 2008). Also in human medicine, a research carried out in a donation center in Crete showed, by flow cytometry, the presence of the parasite in peripheral leukocytes of 33 donors (1.7\%); PCR confirmed the data. In another study carried out in a donation center in the Balearic Islands (Spain), a cryptic infection by $L$. infantum was diagnosed through PCR in the $6 \%$ of HIV-negative donors. The leukodepletion by filtration of the blood was able to remove the parasite from most of the samples (Riera et al. 2008). The availability of quantitative molecular diagnostic techniques, that are more sensitive, rapid, and objective than conventional microscopic methods, makes easier investigation for the occurrence of parasitemia during CanL.

The aim of this study was to evaluate, through qPCR, the prevalence of parasitemia in sick kennel dogs naturally infected by CanL. An evaluation of daily changes of the parasitic load in peripheral blood was also performed.

\section{Materials and methods}

\section{Ethical issues}

The present study was approved by the Ethics Committee of the Istituto Zooprofilattico Sperimentale della Sicilia (Palermo, Italy). All treatments, housing, and animal care were carried out in accordance with the standards recommended by the Companion Animals Protection and Prevention of Straying Animals Law (15/2000) of the Government of Sicily, based on the EU Directive 2010/63/EU for animal experiments. The research was carried out on stray dogs. Dogs were captured by municipal shelter personnel or by authorized volunteers from animal protection associations, as part of a routine procedure for health and reproductive control. The dogs were captured with painless systems and without the use of leghold traps, poisoned baits, or prods. The dogs were housed in a shelter which complied with the maximum number of dogs allowed by Italian law n.15/2000 (400 dogs) and included kennels with an indoor $(3 \mathrm{~m} \times 2 \mathrm{~m})$ and an outdoor $(3 \mathrm{~m} \times$ $4 \mathrm{~m}$ ) section, as required by Italian law.

\section{Animals}

The study was carried out on 140 stray dogs coming from a dog shelter located in Palermo (Sicily, South of Italy), an area highly endemic for CanL, during the period between September 2007 and April 2009.

Animals of different breeds, ages (range between 4 months and 12 years), and genders (59 males and 81 females) were included. On each dog, a general physical examination was performed and blood, conjunctiva, skin, and lymph node samples were collected.

The animals were staged according to Canine Leishmaniasis Working Group (Paltrinieri et al. 2010). All the dogs with clinically evident leishmaniasis were enrolled in the study while exposed and infected dogs were excluded by further evaluations. Dogs with titer $\geq 1: 640$ (fourfold higher than the threshold positive value indicated by the reference laboratory) and/or dogs with positive cytologic results, regardless of serologic or molecular tests (qPCR), were considered sick.

Among sick dogs, those that could undergo blood sampling every $4 \mathrm{~h}$ for a $24-\mathrm{h}$ period were selected, to evaluate possible daily variations of parasitemia. The evaluation of parasitemia was performed on whole blood samples with EDTA using qPCR and by microscopic examination of blood smears.

\section{Sampling}

Blood samples were taken by venipuncture of the cephalic vein and collected in tubes with and without anticoagulant (EDTA) to obtain serum and whole blood, respectively. 
Blood smears were immediately prepared. Popliteal lymph node aspirates were obtained from each dog by using a thin biopsy needle. A thin smear was performed immediately after collection. Biopsy punch ( $2 \mathrm{~mm}$ in diameter) was used to collect a skin specimen from the left shoulder; conjunctival swab samples were obtained from both eyes. The animals enrolled in the chrono-biological study were sampled every $4 \mathrm{~h}$ for 6 times during a 24-h period, at fixed time points (19:00, 23.00, 03:00, 07:00, 11:00, and 15:00). These time points were chosen to have light- and dark-span samples. The samples were kept refrigerated and sent within the next day to the Leishmaniosis National Reference Center of the Istituto Zooprofilattico Sperimentale della Sicilia (Palermo, Italy). The tubes were identified with serial numbers and laboratory operators did not know the dog's identity or the time of sampling.

Whole blood, serum, lymph node, and other samples (conjunctiva and skin) were stored at $-80^{\circ} \mathrm{C}$ until examination.

\section{Indirect fluorescent antibody test}

Indirect fluorescent antibody test (IFAT) was performed using promastigotes of $L$. infantum reference strain IPT1 ZMON1 as antigen. The cells were exposed to sera diluted 1:80 in phosphate-buffered saline (PBS) in a moist chamber, and then to fluoresceinated rabbit anti-dog immunoglobulin $\mathrm{G}$ (IgG) serum diluted $1: 40$ both at $37^{\circ} \mathrm{C}$ for $30 \mathrm{~min}$. Samples were considered positive when promastigote cytoplasmic or membrane fluorescence was observed at a serum dilution of 1:80 (cutoff). Positive sera were titrated until they gave negative results.

\section{Microscopic examination}

Blood and lymph node smears were stained with MayGrünwald Giemsa and examined under an optical microscope to determine whether circulating forms of $L$. infantum were found. Each smear was examined for $10 \mathrm{~min}$ (100 microscopic fields) under a $\times 100$ oil immersion objective lens.

\section{Molecular test}

PCR was performed on whole blood, lymph node, conjunctival swabs, and skin biopsies. Total DNA extraction was performed on lymph node aspirates using "E.Z.N.A. Tissue DNA Kit (Omega biotech VWR)" according to the manufacturer's instructions. The PCR test was targeted on a 123-bp fragment inner to the constant region in the minicircle kinetoplast DNA (kDNA) (NCBI accession number AF291093) and was carried out as previously described (Manna et al. 2006). The primer sequences were as follows: QLK2-U 5'-GGCG TTCTGCGAAAACCG-3'; QLK2-D5' AAAATGGC ATTTTCGGGCC-3'; while the associated probe was as follows: 5'-TGGGTGCAGAAATCCCGTTCA-3' 5'FAM and $3^{\prime}$ BHQ labeled. Each amplification was performed in duplicate, in $20 \mu \mathrm{l}$ reaction mixture containing $1 \times$ TaqMan Universal Master Mix (Applied Biosystem), $20 \mathrm{pmol} / \mu \mathrm{l}$ of the specific primers, and $10 \mathrm{pmol} / \mu \mathrm{l}$ of labeled probe (Qleish 2$), 1 \times \mathrm{EXO}$ IPC Mix, $1 \times$ EXO IPC DNA according to the manufacturer's instructions of the TaqMan Exogenous Internal Positive Control Reagents kit (Applied Biosystem). The thermal cycling conditions comprised an initial incubation for $2^{\prime}$ at $50^{\circ} \mathrm{C}$ for uracil- $\mathrm{N}$ glycosylase activity. This step was followed by a $10^{\prime}$ denaturation at $95^{\circ} \mathrm{C}$ and 45 cycles at $95^{\circ} \mathrm{C}$ for $15^{\prime \prime}$ and $60^{\circ} \mathrm{C}$ for $1^{\prime}$ each. Results were expressed as a parasite count in milliliters of the liquid matrices such as blood, according to the parasite charge per milliliter of the standard curve described below. Standard DNA was extracted from cultured IPT1 MON1, obtained from the collection of the Italian National Reference Centre for Leishmaniasis (C.Re.Na.L), counted at $1 \times 109$ cells $/ \mathrm{ml}$, and homogenized in $1 \mathrm{ml}$ of lysis mix (1\% Tween 20, $1 \%$ Nonidet P-40, e 20\% Chelex). Then, decimal serial dilutions of the stock solution were performed to obtain the points of the curve ranging from the DNA equivalent of $1 \times$ 106 cells to 1 cell in milliliters (Vitale et al. 2004; Manna et al. 2006). The sensitivity of the qPCR assays designed on minicircle kDNA conserved regions was tested by using serial dilutions of parasite DNA extracted from a known number of parasites. Detection of the kinetoplast DNA of $L$. infantum reached the level of 0.0005 parasites per reaction tube with a dynamic range of 107 (Mary et al. 2004.).

The dogs with parasitemia were also tested for endemic canine vector-borne diseases (CVBD): Ehrlichia canis, Anaplasma phagocytophilum, Rickettsia conorii, Babesia canis (IFI and PCR), and microfilaria (Knott test).

\section{Statistical analysis}

Data were tested with the Shapiro-Wilk normality test. Data did not result to be normally distributed $(p>0.05)$ and were analyzed using the Mann-Whitney $U$ test and the Kolmogorov-Smirnov test for non-parametric analysis, in order to compare the medians of blood and lymph node parasite loads in dogs with parasitemia. The Pearson test was performed to assess significant correlations between the blood and lymph node parasite loads in positive dogs with parasitemia. A $p$ value $<0.05$ was considered statistically significant. Statistical analysis was performed using the STATISTICA software package (STATISTICA 7, Stat Software Inc., Tulsa, Oklahoma).

\section{Results and discussion}

Among the 140 dogs enrolled in the present study, 39 (28.1\%) were diagnosed to be sick dogs with clinically evident 
leishmaniasis based on serological, molecular, and microscopic findings (Table 1). These dogs were adult (age range: 212 years), of different breeds and genders (14 males and 25 females) and showed various clinical signs consistent with the disease. Twelve $(30.8 \%)$ out of the 39 sick dogs showed parasitemia, with a load ranging from 1 to 10 Leishmania/ml
Table 1 Positivity to each diagnostic techniques of 39 sick dogs with clinically evident leishmaniasis (dogs $31-37$ reported in Table 2 are the same)

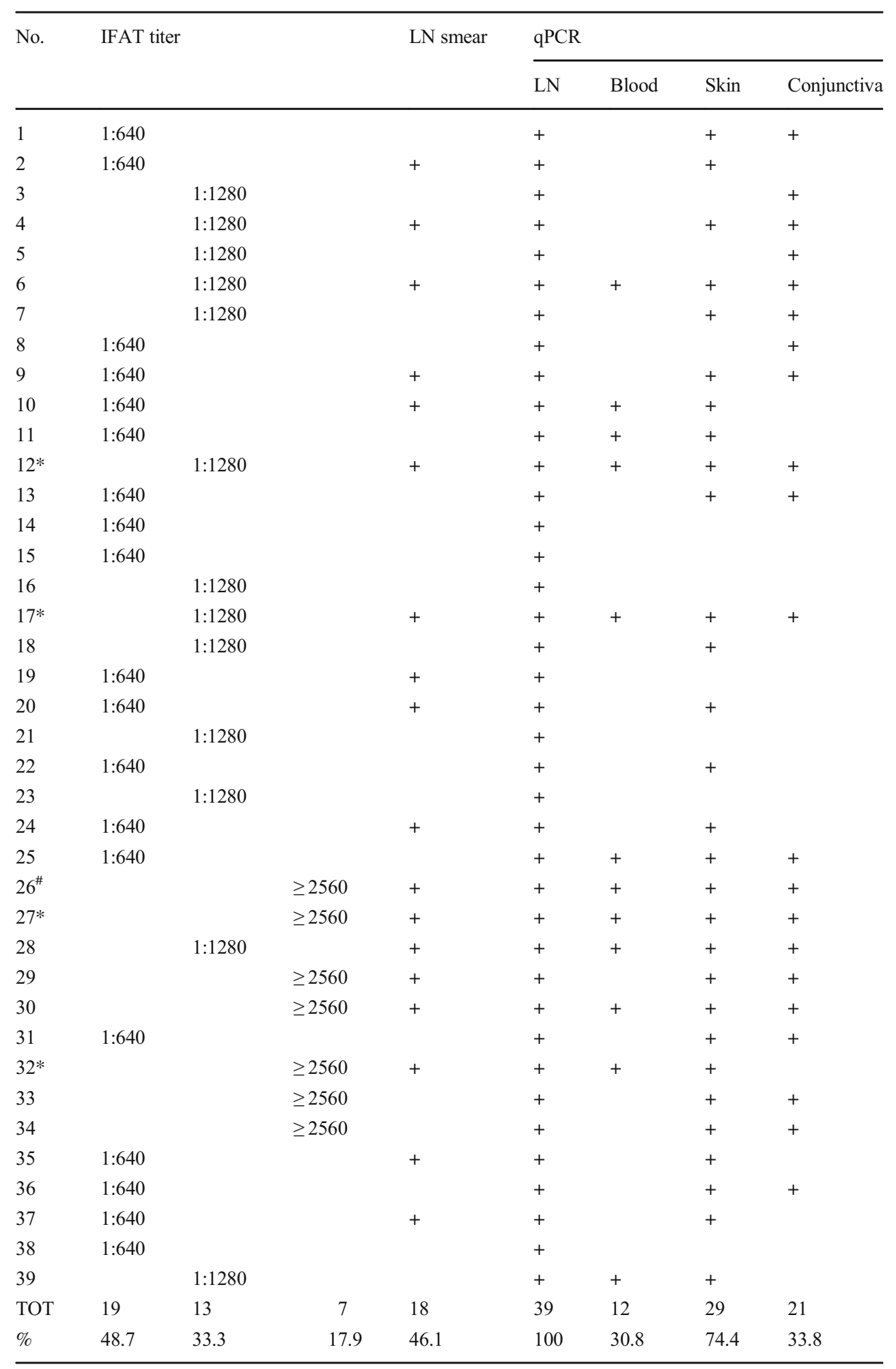

$L N$, lymph node; +, positive; IFAT, immunofluorescence antibody test; $q P C R$, quantitative polymerase chain reaction. ${ }^{*}$ Coinfection with E. canis; ${ }^{\#}$ Coinfection with E. canis, A. phagocytophilum, B. canis, R. conorii, and Acanthocheilonema reconditum 
(median: 4). The amount of Leishmania DNA found in blood samples was lower than in lymph nodes. In fact, the lymph node parasite load ranged from 4 to10,000,000 Leishmanial $\mathrm{ml}$ (median: 4000). Statistically significant difference in the medians of DNA quantity was found among the different biological samples (blood and lymph node) $(p<0.001)$ (Fig. 1). The Pearson test showed no significant correlation between blood and lymph node parasite loads $(p=0.96)$ of the twelve positive dogs with parasitemia. Surprisingly, Leishmania DNA was also detected in the peripheral blood of asymptomatic dogs, negative to both serology and PCR performed on samples other than blood $(6 / 101 ; 5.9 \%)$. The blood parasite load ranged from 5 to 80 Leishmania/ml (median: 20). Microscopic examination of peripheral blood smears revealed no amastigote forms in any samples at any time. Out of twelve parasitemic dogs, five were co-infected with one or more CVBD (Table 1). A serial blood sampling was possible only on 7 sick dogs (3 males and 4 females; age range: $2-8$ years). The lymph node parasite load in these dogs ranged from 7000 to 1000000 Leishmania/ml (median: 30000). IFAT titers ranged from 1:640 to 1:2560. A total of 42 blood samples were obtained. Among the serially sampled dogs, only one out of 7 (14.3\%) showed Leishmania DNA in the peripheral blood by qPCR. In this dog, parasitemia was recorded only in two out of the $6(33.3 \%)$ samples collected during the $24-\mathrm{h}$ period, corresponding to $4.8 \%$ (2/42) of all the samples. The positive blood samples were collected at time points 11:00 and 15:00 and they showed a very low parasitic
Fig. 1 Descriptive statistic (median, minimum [Min], maximum [Max], 25\% and $75 \%$ percentiles) of blood and lymph node loads (no. of parasites $/ \mathrm{mL}$ ) of the 12 dogs affected by canine leishmaniasis (CanL) which showed parasitemia Leishmania
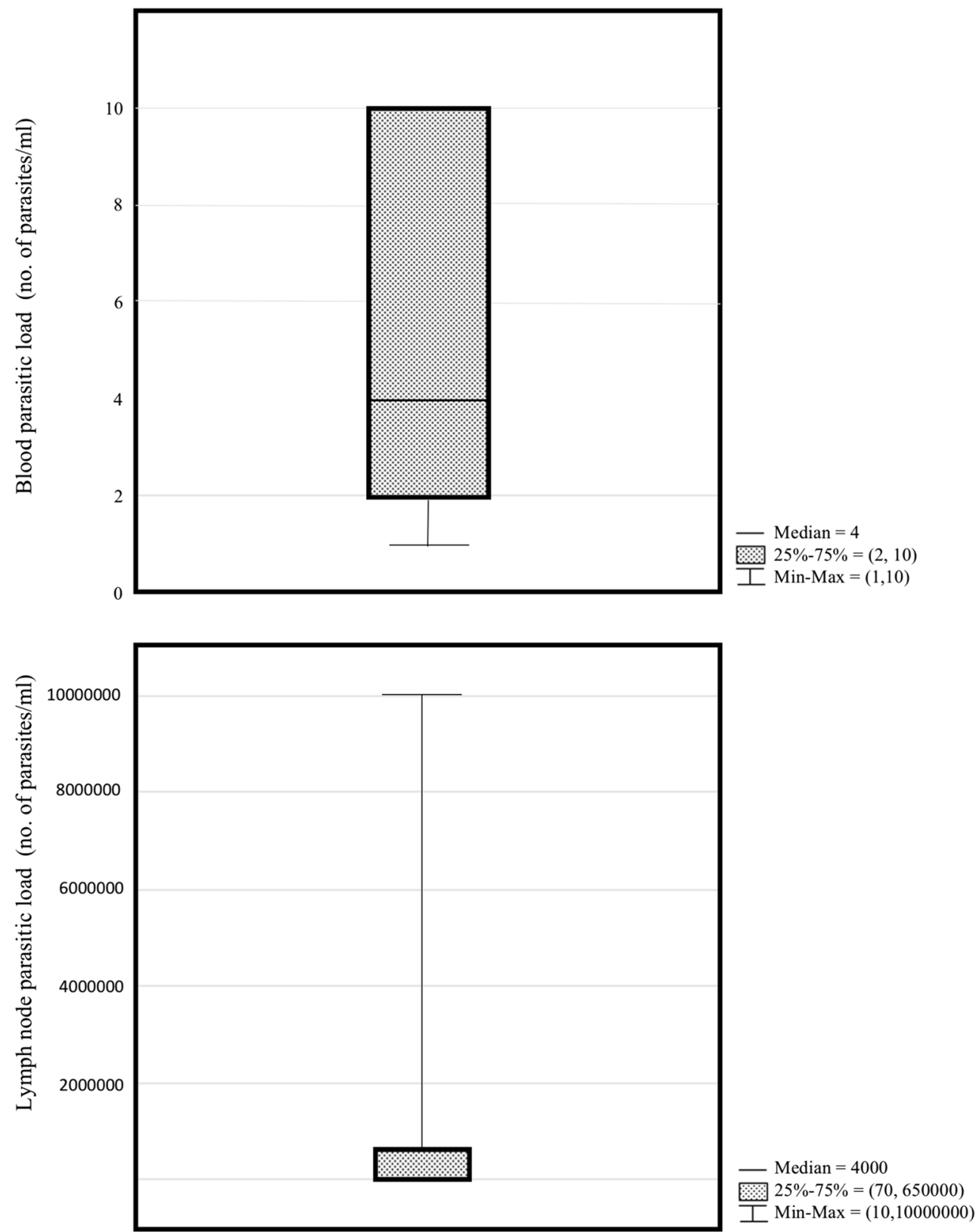
load (4 and 1 Leishmania/ml, respectively). Information regarding clinical signs, blood, and lymph node parasite loads and IFAT titers of the 7 dogs serially sampled are reported in Table 2.

The present report confirmed that during CanL the presence of parasitemia is infrequent $(31 \%)$ and often observed in co-infected dogs $(5 / 12,41.7 \%)$, as observed in previous studies (Giudice and Passantino 2011; Manna et al. 2004; Oikonomidis et al. 2019). In this study, we have deliberately chosen to evaluate sick dogs with clinically evident leishmaniasis, in order to know the real prevalence, intensity, and constancy of parasitemia during the disease, excluding exposed and infected dogs which could underestimate the results. Our findings suggest that the hematogenous dissemination occurs only in a small number of sick animals, confirming that the routine screening of blood samples, through PCR and smear microscopy, must not be currently advised to establish a diagnosis, because of lower diagnostic sensitivity compared with bone marrow or lymph node samples (Sharma et al. 2000; Delgado et al. 1998; Oikonomidis et al. 2019); the sensitivity increases performing a buffy coat (Oikonomidis et al. 2019). Furthermore, the blood parasite load was very low even in dogs with high lymph node parasite loads and high IFAT titers, in accordance with other reports describing natural infections (Manna et al. 2008; 2006; 2004). The lack of correspondence between lymph node and blood had already been observed in previous studies performed with microscopic methods in humans affected by Kala-azar due to Leishmania donovani (Sharma et al. 2000; Saran et al. 1997). Interestingly, our findings seem to suggest that the presence of parasitemia is transitory, since it was not constantly observed in the same animal. However, the results of the chrono-biological study are preliminary, due to the small number of enrolled animals, given from the difficulty to find untreated sick dogs on which serial withdrawals over $24 \mathrm{~h}$ were performed. A chrono-biological statistical analysis was not performed because there was only a dog with positive blood. The dog had a very low parasitic load in only two time points, both during the daytime. This would seem in disaccordance with the Kala-azar disease, in which the maximum of parasitemia was recorded during the night, at the peak of sandflies' activity (Sharma et al. 2000; Saran et al. 1997). Further studies should be carried out on a larger sample of dogs affected by leishmaniasis throughout the whole year, in order to confirm these preliminary results. It would also be interesting to assess the degree of parasitemia in relation to light-dark alternation and to evaluate different sampling sites and methods, in order to improve diagnostic accuracy. Although it was hypothesized that the blood acts as a medium for parasite transport rather than a reservoir tissue and that the parasite quantification in blood samples appears not significant in determining the course of infection (Manna et al. 2006), the detection of Leishmania DNA in peripheral blood of six negative asymptomatic dogs $(6 / 101 ; 5.9 \%)$ is very interesting and clinically relevant. These findings agree with previous surveys carried out on blood donors in endemic areas, both in humans and in animals (Riera et al. 2008; Tabar et al. 2008; de Freitas et al. 2006; Kyriakou et al. 2003; Otero et al. 2000) and imply a transfusion risk, regardless of the tests carried out. A reduction in risk could arise by using leukocyte filtration in endemic areas (Riera et al. 2008). Another implication is the possible transmission of the
Table 2 Main clinical findings, IFAT titer, and parasite load of blood and lymph node samples from 7 serial sampled dogs naturally infected by $L$. infantum

\begin{tabular}{|c|c|c|c|c|c|c|c|}
\hline \multirow[t]{2}{*}{ Clinical findings } & \multicolumn{7}{|l|}{ Dog } \\
\hline & 1 & 2 & 3 & 4 & 5 & 6 & 7 \\
\hline Depression & + & & & + & + & + & + \\
\hline Anorexia/dysorexia & + & & & & & + & + \\
\hline Weight loss & + & & & & & + & + \\
\hline Pale mucous membranes & + & & & & & + & + \\
\hline Onychogryphosis & & & & & + & & \\
\hline Dermatitis/alopecia & + & & + & & + & & + \\
\hline Lymphadenopathy & + & + & + & + & + & + & + \\
\hline Splenomegaly & + & + & & & & & \\
\hline Blepharitis & + & + & + & + & & + & \\
\hline Ocular lesions & & + & & + & + & & \\
\hline Buccal ulcerations & & & + & & & & \\
\hline IFAT & $1: 640$ & $1: 2560$ & $1: 2560$ & $1: 2560$ & $1: 640$ & $1: 640$ & $1: 640$ \\
\hline Blood qPCR (Leishmania/ml) & - & 10 & - & - & - & - & - \\
\hline $\begin{array}{l}\text { Lymph node qPCR } \\
\text { (Leishmania/ml) }\end{array}$ & 7000 & $1,000,000$ & 10,000 & 30,000 & 300,000 & 10,000 & $1,000,000$ \\
\hline
\end{tabular}


Leishmania spp. to sandfly even by seronegative asymptomatic animals, which makes very important the constant use of repellents on both client-owned and kennel dogs.

Acknowledgments The authors thank the municipal shelter personnel in Palermo and the IZS personnel. The authors are grateful to Ms. Caroline Keir for assistance with language and for her careful review of the manuscript.

\section{Compliance with ethical standards}

All treatments, housing, and animal care were carried out in accordance with the standards recommended by the Companion Animals Protection and Prevention of Straying Animals Law (15/2000) of the Government of Sicily, based on the EU Directive 2010/63/EU for animal experiments. The present study was approved by the Ethics Committee of the Istituto Zooprofilattico Sperimentale della Sicilia (Palermo, Italy).

Conflict of interest The authors declare that they have no conflict of interest.

Abbreviations CanL, canine leishmaniasis; IFAT, indirect fluorescent antibody test; IgG, immunoglobulin G; PBS, phosphate-buffered saline; qPCR, quantitative polymerase chain reaction

\section{References}

Alvar J, Vélez ID, Bern C, Herrero M, Desjeux P, Cano J, Jannin J, den Boer M (2012) The WHO Leishmaniasis Control Team. Leishmaniasis worldwide and global estimates of its incidence. PLoS One 7:e35671

Anand M, Kumar R, Malhotra OP, Singh S (2004) Amastigote forms of Leishmania donovani in peripheral blood. Indian J Pathol Microbiol 47:307

Chemli J, Abroug M, Fathallah A, Abroug S, Ben Said M, Harbi A (2006) Contribution of leukoconcentration in the diagnosis of Kala-azar in Tunisia. Méd Mal Infect 36:390-392

de Freitas E, Melo MN, da Costa-Val AP, Michalick MS (2006) Transmission of Leishmania infantum via blood transfusion in dog: potential for infection and importance of clinical factors. Vet Parasitol 137:159-167

Delgado J, Pineda JA, Macías J, Regordán C, Gallardo JA, Leal M, Sanchez-Quijano A, Lissen E (1998) Low sensitivity of peripheral a. blood smear for diagnosis of subclinical visceral leishmaniasis in human immunodeficiency virus type 1-infected patients. J Clin Microbiol 36:315-316

Fiorini M, Messina MF, Barracchia A (2002) Peripheral intramonocytic and intraneutrophil leishmanias observed in a chronic myelomonocytic leukemia (CMMoL) patient. Haematologica 87 : EIM21

Foglia Manzillo V, Piantedosi D, Cortese L (2005) Detection of Leishmania infantum in canine peripheral blood. Vet Rec 156: 151-152

Giger U, Oakley DA, Owens SD, Schantz P (2002) Leishmania donovani transmission by packed RBC transfusion to anemic dogs in the United States. Transfusion 42:381-383

Giudice E, Passantino A (2011) Detection of Leishmania amastigotes in peripheral blood from four dogs-short communication. Acta Vet Hung 59:205-213
Izri MA, Deniau M, Brière C, Rivollet D, Pethitory JC, Houin R, Rousset JJ (1996) Leishmaniosis in AIDS patients: results of leukocyteconcentration a fast biological method of diagnosis. Bull WHO 74:91-93

Kyriakou DS, Alexandrakis MG, Passam FH, Kourelis TV, Foundouli P, Matalliotakis E, Maniatis AN (2003) Quick detection of Leishmania in peripheral blood by flow cytometry. Is prestorage leucodepletion necessary for leishmaniasis prevention in endemic areas? Transfus Med 13:59-62

Maia C, Campino L (2008) Methods for diagnosis of canine leishmaniasis and immune response to infection. Vet Parasitol 158:274-287

Manna L, Vitale F, Stefano R, Caracappa S, Pavone ML, Della Morte R, Cingoli G, Staiano N, Gravino AE (2004) Comparison of different tissue for PCR-based diagnosis and follow-up of canine visceral leishmaniosis. Vet Parasitol 125:251-261

Manna L, Reale S, Viola E, Vitale F, Foglia Manzillo V, Pavone LM, Caracappa S, Gravino AE (2006) Leishmania DNA load and cytokine expression levels in asymptomatic naturally infected dogs. Vet Parasitol 142:271-280

Manna L, Reale S, Vitale F, Picillo E, Pavone LM, Gravino AE (2008) Real-time PCR assay in Leishmania infected dogs treated with meglumine antimoniate and allopurinol. Vet J 177:279-282

Mary C, Faraut F, Lascombe L, Dumon H (2004) Quantification of Leishmania infantum DNA by a real-time PCR assay with high sensitivity. J Clin Microbiol 42:5249-5255

Mendoza-Roldan J, Benelli G, Panarese R, Iatta R, Furlanello T, Beugnet F, Zatelli A, Otranto D (2020) Leishmania infantum and Dirofilaria immitis infections in Italy, 2009-2019: changing distribution pattern. Parasites Vectors 13(1):193. https://doi.org/10.1186/s13071-02004063-9

Nandy A (1986) Parasitemia in Indian Kala-azar. Ann Trop Med Parasitol $80: 463-464$

Oikonomidis IL, Tsouloufi TK, Mylonakis ME, Psalla D, Soubasis N, Rallis T, Kritsepi-Konstantinou M (2019) Circulating and bone marrow myeloid cells containing Leishmania amastigotes in a case of advanced canine leishmaniosis. J Vet Diagn Investig 31(5):726-731

Otero AC, da Silva VO, Luz KG, Palatnik M, Pirmez C, Fernandes O, Palatnik de Sousa CB (2000) Short report: occurrence of Leishmania donovani DNA in donated blood from seroreactive Brazilian blood donors. Am J Trop Med Hyg 62:128-131

Owens SD, Oakley DA, Marryott K, Hatchett W, Walton R, Nolan TJ, Newton A, Steurer F, Schantz P, Giger U (2001) Transmission of visceral leishmaniasis through blood transfusions from infected English foxhounds to anemic dogs. J Am Vet Med Assoc 15: 1076-1083

Paltrinieri S, Solano-Gallego L, Fondati A, Lubas G, Gradoni L, Castagnaro M, Crotti A, Maroli M, Oliva G, Roura X, Zatelli A, Zini E (2010) Guidelines for diagnosis and clinical classification of leishmaniasis in dogs. J Am Vet Med Assoc 236(11):1184-1191

Ramos RA, Ramos CA, Santos EM, de Araújo FR, de Carvalho GA, Faustino MA, Alves LC (2013) Quantification of Leishmania infantum DNA in the bone marrow, lymph node and spleen of dogs. Rev Bras Parasitol Vet 22:346-350

Riera C, Fisa R, López-Chejade P, Serra T, Girona E, Jiménez M, Muncunill J, Sedeño M, Mascaró M, Udina M, Gállego M, Carrió J, Forteza A, Portús M (2008) Asymptomatic infection by Leishmania infantum in blood donors from the Balearic Islands (Spain). Transfusion 48:1383-1389

Ruiz De Gopegui R, Espada Y (1998) Peripheral blood and abdominal fluid from a dog with abdominal distention. Vet Clin Pathol 27:64 67

Saran R, Sharma MC, Gupta AK, Sinha SP, Kar SK (1997) Diurnal periodicity of Leishmania amastigotes in peripheral blood of Indian Kala-azar patie. Acta Trop 68:357-360 
Sharma MC, Gupta AK, Das VNR, Verma N, Kumar N, Saran R, Kar SK (2000) Leishmania donovani in blood smears of asymptomatic persons. Acta Trop 76:195-196

Tabar MD, Roura X, Francino O, Altet L, Ruiz de Gopegui R (2008) Detection of Leishmania infantum by real-time PCR in a canine blood bank. J Small Anim Pract 49:325-328
Vitale F, Reale S, Vitale M, Petrotta E, Torina A, Caracappa S (2004) TaqMan-based detection of Leishmania infantum DNA using canine samples. Ann N Y Acad Sci 1026:139-143

Publisher's note Springer Nature remains neutral with regard to jurisdictional claims in published maps and institutional affiliations. 